auftragten Nebenklagevertreterinnen oder Nebenklagevertreter für Unterstützung, Begleitung, Erklärung und Trost zur Verfügung. Hauptaufgabe dieses juristischen Beistandes ist jedoch, die Wahrung der Beteiligungs- und Informationsrechte der kindlichen und jugendlichen Verletzten zu gewährleisten. Nebenklageanwältin und Nebenklageanwalt müssen an der Hauptverhandlung zur Wahrung der Rechte ihrer Mandantinnen und Mandanten von Beginn an teilnehmen, insbesondere auch während der Einlassung der oder des Angeklagten zur Person oder zur Sache. Zwar haben auch die Verletzten das Recht, selbst an der gesamten Beweisaufnahme, also auch während der Aussage der oder des Angeklagten, anwesend zu sein. Aus Gründen ihrer Glaubwürdigkeit ist dies jedoch regelmäßig kontraproduktiv. Warten sie indes ihren Aufruf als Zeugin oder Zeuge außerhalb des Gerichtssaals ab, stehen ihnen Nebenklagevertreterin oder Nebenklagevertreter für ihre Begleitung nicht zur Verfügung. Vor allem aber sind die Nebenklageanwältin und der Nebenklageanwalt für sozialpädagogische oder psychosoziale Begleitung bei potentiell traumatisierten Zeuginnen oder Zeugen zumeist nicht ausgebildet. Die Aufgabe muss deshalb nach dem Ergebnis der Diskussion Prozessbegleiterinnen oder Prozessbegleitern übertragen werden. ${ }^{2}$ Dabei wurde aber zugleich festgestellt, dass es der Erarbeitung von Standards für die Durchführung der Prozessbegleitung ebenso bedarf wie für die Durchführung der Aus- bzw. Fortbildung dieser Prozessbegleiterinnen und Prozessbegleiter. Zudem wird zu prüfen sein, ob das Angebot sozialpädagogischer oder psychosozialer Prozessbegleitung nur bestimmten Gruppen von Opfern angeboten werden sollte, wie zum Beispiel kindlichen und jugendlichen Opfern von Straftaten oder generell Opfern von Gewalttaten oder besonders schweren Straftaten. Wie schlussendlich eine gesetzliche Verankerung in der Strafprozessordnung erfolgen kann, wird im Hinblick auf die Frage der Kostenübernahme durch den Staat oder durch den Verurteilten noch weiter zu diskutieren sein.

Siehe auch Stellungnahme des Deutschen Juristinnenbundes (djb)
St 07-21 Psychosoziale Prozessbegleitung unter www.djb.de/ stellungnahmen-und-pressemitteilungen/.

\title{
Nebenklage: im Sinne der Opfer und der Wahrheitsermittlung
}

\author{
Bewertung zweier aktueller Gesetzentwürfe
}

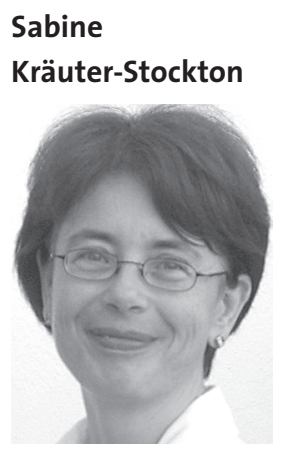

Mitglied der Kommission Gewalt gegen Frauen und Kinder des djb; Vorsitzende des Landesverbands Saarland im djb; Staatsanwältin im Sonderdezernat Häusliche Gewalt und Sexuelle Gewalt, zuvor Koordinierungsstelle gegen Häusliche Gewalt beim Saarländischen Justizministerium, Saarbrücken
Seit Jahrzehnten versteht es der djb als seine Aufgabe, das Augenmerk auf die - heute nach wie vor bestehende - Notwendigkeit zu lenken, die Stellung von Zeuginnen und Zeugen, insbesondere von Verletzten im Strafverfahren zu verbessern ${ }^{1}$. Das Thema ist jetzt wieder einmal hoch aktuell: Im Bundesrat werden zwei Gesetzentwürfe diskutiert, die unter dem Gesichtspunkt des Zeugenschutzes eingebracht wurden. In dem einen wird vorgeschlagen, die Nebenklagebefugnis mit der Möglichkeit einer kostenlosen Opferanwältin oder eines kostenlosen Opferanwalts auf Opfer von Zwangsheirat und von schwerem „Stalking“ zu erstrecken ${ }^{2}$, der andere will die Beiordnung einer Opferanwältin oder eines Opferanwalts für die bereits nach jetziger Rechtslage nebenklageberechtigten Opfer von schweren Körperverletzungen und von erpresserischem Menschenraub sowie von Geiselnahme ermöglichen ${ }^{3}$.

\section{Warum Zeugenschutz?}

Das Erfordernis des Zeugenschutzes folgt grundsätzlich aus der staatlichen Schutzpflicht gegenüber Beeinträchtigungen von Leben und körperlicher Unversehrtheit, aus der Pflicht zur Achtung der Per- sönlichkeit und der gerichtlichen und staatsanwaltschaftlichen Fürsorgepflicht für Zeuginnen und Zeugen ${ }^{4}$. Zeugenschutz war zwar schon in den Anfängen der deutschen Prozessrechtskodifizierung ein Thema ${ }^{5}$, wurde aber erst in den 1980er Jahren Gegenstand eingehender kriminalpolitischer Erörterungen, die einen Perspektivwechsel hinsichtlich der Stellung von Zeuginnen und Zeugen und insbesondere von Opferzeuginnen und Opferzeugen im

1 U.a. Stellungnahme zum Entwurf eines Ersten Gesetzes zur Verbesserung der Stellung von Verletzten im Strafverfahren vom 15.5.1986 (Archiv des djb); Fachtagung des djb vom 30.9.-2.10.1994 in der Berliner Humboldt-Universität zur Verbesserung der Situation von Opfern sexueller Gewalt; Fachtagung des djb in Bonn vom 27.2.1998 zur Reform der Nebenklage; Nelles, U./Oberlies, D.: Reform der Nebenklage und anderer Verletztenrechte, Nomos 1998.

2 BR Drs 872/07.

3 BR Drs 656/07.

4 Löwe/Riess: Die StPO und das GVG, Großkommentar, Einleitung Abschn. I Fn 127.

5 Zeugenschutz wurde schon im 19. Jahrhundert in den Beratungen zur Einführung der StPO diskutiert; siehe hierzu: Christina Ullrich, Schutz des verletzten Zeugen durch Entfernung des Angeklagten gem. § 247 StPO im Bereich der Sexualdelinquenz, LIT-Verlag Berlin-Hamburg-Münster 1998, S. 19-21. 
Strafverfahren postulierten und mehrere Gesetzesänderungen zur Folge hatten ${ }^{6}$. Als besonders schutzbedürftig werden von jeher die durch die Straftat verletzten Opferzeuginnen und Opferzeugen angesehen, die nicht selten durch das Gerichtsverfahren zum zweiten Mal traumatisiert zu werden drohen (Sekundärviktimisierung): als einzige oder als Hauptbelastungszeugen unterliegt ihre Aussage oft einer besonders eingehenden Glaubhaftigkeitsprüfung, werden sie immer wieder nach den schmerzlichen Einzelheiten der Tat befragt und können sich gegen unberechtigte Schuldzuweisungen seitens des Angeklagten oft nur unzureichend wehren.

\section{Verbesserungen durch Nebenklage}

Durch das Zeugenschutzgesetz von $1998^{7}$, die vorerst letzte größere Veränderung auf dem Gebiet des Zeugenschutzes, war mit $\$ 68 \mathrm{~b}$ StPO die Bestellung eines Zeugenbeistands während einer Vernehmung und mit $\$ 397$ a StPO ein solcher für bestimmte Nebenklagefälle eingeführt worden. Diese verfahrensrechtliche Änderung hat sich in der Praxis bewährt. Durch die Zulassung der Nebenklage der verletzten Person und die Beiordnung eines anwaltlichen Beistands wird nicht nur ihre Situation spürbar erleichtert. Hierdurch wird auch umgesetzt, was das Bundesverfassungsgericht vor 30 Jahren anmahnte, dass es nämlich dem Gebot des fairen Verfahrens entspricht, Bürgerinnen und Bürgern, die in ein justizförmiges Verfahren eingebunden werden, bei der Wahrnehmung prozessualer Rechte anderen Verfahrensbeteiligten gegenüber eine verfahrensrechtliche Selbstständigkeit zu gewähren ${ }^{8}$.

\section{Pro und contra Nebenklage}

Der Befürwortung des Instituts der Nebenklage und der damit verbundenen Rechte für die Opferzeuginnen/-zeugen wird teilweise entgegengehalten, die über allem justiziellen Handeln stehende Pflicht zur Wahrheitsermittlung könnte durch damit einhergehende Maßnahmen wie etwa Akteneinsichtnahme gefährdet werden - sei doch dann die Zeugenaussage nicht mehr spontan, möglicherweise von taktischen Überlegungen geleitet und damit nicht mehr zuverlässig der gerichtlichen Sachverhaltswürdigung zugrunde zu legen ${ }^{9}$. Diese Polarisierung ist jedoch nicht begründet. Was nützt der Wahrheitsfindung etwa eine Zeugin, die sich ohne anwaltlichen Beistand aus Angst und vor Aufregung in der Prozesssituation nicht mehr an ihre Erlebnisse erinnern kann oder die gar aus Unsicherheit, um das Gericht von dem ihr durch den Angeklagten zugefügten Unrecht zu überzeugen, noch ein paar Kleinigkeiten dazu „dichtet“? Dies alles kennen wir in der Praxis zur Genüge, um sagen zu können, dass die nicht an- waltlich vertretenen Zeuginnen und Zeugen beileibe nicht die „besseren“ Zeuginnen oder Zeugen sind - im Gegenteil! Es ist eine Illusion anzunehmen, Zeugenerinnerungen könnten ohne juristische oder sonstige Beratung gleichsam unter einer Glasglocke frisch gehalten und dann wie eine Filmaufnahme nach Monaten vor Gericht abgespult werden. Die Erinnerung aller Zeuginnen und Zeugen unterliegt zahlreichen sowohl intentionalen als auch unbeabsichtigten Einflüssen ${ }^{10}$. Die Unzuverlässigkeit der Zeugenaussagen wird dann noch weiter verstärkt, wenn die aussagende Person in der ungewohnten Situation vor Gericht Angst hat und vielleicht neben ihrer Erinnerungsleistung auch noch darauf achten muss, dass sie nicht zuviel von sich preisgibt, um dem Angeklagten oder ihm nahe ste-

\section{Im Bewusstsein, dass durch die anwaltliche Vertretung für die Wahrung der eigenen Rechte gesorgt wird, kann sich die Zeugin oder der Zeuge auf die Erinnerungsleis- tung konzentrieren und damit optimal zur Wahrheits- findung beitragen.}

hendem Publikum im Gerichtssaal keine Ansatzpunkte für Rachemaßnahmen zu bieten. Hier finden sich zwei wichtige Aufgabenfelder für die anwaltliche Vertretung. In dem beruhigenden Gefühl, nicht allein sondern fachkundig vertreten vor Gericht zu stehen und im Bewusstsein, dass durch die anwaltliche Vertretung für die Wahrung der eigenen Rechte gesorgt wird, kann sich die Zeugin oder der Zeuge auf die Erinnerungsleistung konzentrieren,

6 Das Opferschutzgesetz (1986): Ausgestaltung der Nebenklage als Schutzinstrument für in höchstpersönlichen Rechtsgütern Verletzte ( $\$ 395$ StPO, Beteiligungsbefugnisse und Informationsrechte für Opfer von Straftaten ( $\$ \S 406 d-406 \mathrm{~h}$ StPO), unter bestimmten Voraussetzungen Möglichkeit des Ausschlusses des Angeklagten während der Zeugenvernehmung zur Vermeidung unzumutbarer Konfrontation von Zeugen und Angeklagten ( $\$ 247$ StPO), Ausschluss der Öffentlichkeit während der Erörterung von Umständen aus dem persönlichen Lebensbereich der Zeugen ( $\$ 171 b$ GVG neu), Erweiterung der Möglichkeit, Fragen an Zeugen als unzulässig zurückzuweisen, die ohne Not deren persönlichem Lebensbereich betreffen ( $\$$ 68a StPO), Änderungen im Adhäsionsverfahren zur Erleichterung der Schadenswiedergutmachung ( $\$ \S$ 403, 404, 406 StPO).

Das Zeugenschutzgesetz (1998): Möglichkeit der Videoaufzeichnung einer Zeugenvernehmung außerhalb der Hauptverhandlung und Vorführung der Aufnahme in der Hauptverhandlung, Videovernehmung außerhalb des Sitzungszimmers mit Übertragung in den Sitzungssaal ( $\$ \$ 58 a, 255 a, 247 a$ StPO).

7 Siehe oben Fn. 6.

8 BVerfGE 38, 105, 112.

9 Christine Horn: Im Namen der Opfer, Novo-Magazin April 1999.

10 S. Luise Greuel u.a.: Glaubhaftigkeit der Zeugenaussage, Beltz Psychologie Verlags Union, Weinheim, m.w.N. 
wird unabgelenkt eine bestmögliche Aussage machen und damit optimal zur Wahrheitsfindung beitragen können. Die Besorgnis einer Beeinträchtigung der richterlichen Würdigung der Zeugenaussage durch „Einpauken“ der Erstaussage - was theoretisch der anwaltlichen Vertretung mittels Akteneinsicht möglich wäre - besteht nur im Fall eines unprofessionellen Vorgehens der Nebenklagevertretung ${ }^{11}$. Die Zulassung der Nebenklage mit für das Opfer kostenloser - anwaltlicher Vertretung liegt damit nicht nur im Interesse des Opfers, sondern auch dem der Wahrheitsfindung. Kostenlos muss Nebenklagevertretung deshalb sein, weil der wegen der Straftat verurteilte Täter, der zum Tragen der Nebenklagekosten verurteilt wird, häufig nicht

\section{Die Zulassung der Nebenklage mit - für das Opfer kostenloser - anwaltlicher Vertretung liegt damit nicht nur im Interesse des Opfers, sondern auch dem der Wahrheitsfindung.}

über die notwendigen finanziellen Mittel zur Kostendeckung verfügt, so dass das Opfer schlussendlich selbst das Kostenrisiko trägt. Eine Rechtsschutzversicherung, wenn denn eine solche für das Opfer besteht, lehnt in der Regel Kostentragung für eine Nebenklage ab. In diese Richtung zielen die beiden eingangs erwähnten aktuellen Gesetzentwürfe im Bundesrat, die deshalb zu unterstützen sind.

\section{Änderung des $\$ 395$ StPO}

Opfer von Zwangsverheiratung ( $\mathbb{2} 240$ Abs. 4 Z. 1 Alt. 2 StGB) sind durch die Folgen der erlittenen Straftat oftmals lebenslang beeinträchtigt, eigenverantwortliches Entscheiden und Handeln im Kontext des Strafverfahrens fällt ihnen, da nicht eingeübt, unverhältnismäßig schwer. Sie fürchten die Konfrontation mit dem Täter und dessen Clan und sind dringend auf professionelle anwaltliche Unterstützung angewiesen. Hier setzt der Gesetzentwurf 872/07 an, der vorschlägt, Zwangsverheiratungsopfern eine Nebenklage zu erlauben, indem der $\mathbb{} 240$ Abs. 4 Z. 1 Alt. 2 StGB in den Katalog der nebenklagefähigen Delikte des $\mathbb{} 395$ StPO aufgenommen wird.

\section{Weitergehende Forderung: $§ 240$ Abs. 4 Z. 1 Alt. 1 StGB}

Der unterstützenswerte Vorstoß geht jedoch nicht weit genug. Opfer von Nötigung zu einer sexuellen Handlung gem. $\$ 240$ Abs. 4 Z. 1 Alt. $1 \mathrm{StGB}^{12}$ sind in gleicher Weise in ihrer Lebensgestaltung, ihrem Selbstwert und der Fähigkeit zu eigenverantwortlichem Handeln beeinträchtigt wie Opfer von sexuellen Nötigungen i.S. des $\mathbb{} 177$ StGB und anderen
Nebenklagedelikten. Somit ist hier angeraten, bei dieser Gelegenheit auch den $\mathbb{} 240$ Abs. 4 Z. 1 Alt. 1 StGB in den Katalog des $\$ 395$ StPO einzureihen.

\section{Änderung des § $397 a$ Abs. 1 StPO}

In vergleichbarer psychischer Situation wie die genannten Personenkreise befinden sich die Opfer schwerer Körperverletzung (\$ 226 StGB), erpresserischen Menschenraubs ( $\mathbb{S} 239$ a StGB) oder von Geiselnahme ( $\$ 239$ b StGB), die bereits nach derzeitiger Gesetzeslage nebenklagebefugt sind. Es ist ihnen somit zwar möglich, Nebenklage zu erheben. Die Opfer tragen jedoch das Kostenrisiko, weil der Staat nicht eintritt, was die Verletzten nicht selten davon abschreckt, sich anwaltlichen Beistands zu bedienen. Es wurde bereits darauf hingewiesen, dass eine anwaltliche Vertretung in der Nebenklage nicht nur für das Wohl der aussagenden Person, sondern auch für die Wahrheitsfindung von großem Wert ist. Es sind daher die beiden genannten Gesetzentwürfe mit der ausgeführten Erweiterung betreffend Nötigung zu einer sexuellen Handlung zu unterstützen, wonach die $\mathbb{S} \mathbb{S} 226,239 \mathrm{a}, 239 \mathrm{~b}, 240$ Abs. $4 \mathrm{Z}$. 1 (beide Alternativen) StGB in den Katalog des \397a Abs. 1 StPO einzufügen sind. Damit entsteht die Möglichkeit eines für die Opfer solcher Delikte kostenfreien anwaltlichen Beistands.

\section{Kosten}

Der Ablehnung der Forderung unter Hinweis auf die leeren Kassen der Länderhaushalte steht entgegen, dass nicht für alle Geschädigten und nicht in allen Fällen anwaltliche Unterstützung zu bezahlen sein wird. Die Kosten für die Nebenklage werden nach $\mathbb{3}$ 397a StPO nur dann dem Staat überbürdet, wenn das Opfer „seine Interessen ersichtlich selbst nicht ausreichend wahrnehmen “ kann ( $\mathbb{S} 397$ a Abs. 1 S. 2 StPO). Dies hat das Gericht zu prüfen, so dass in Bagatellfällen Ansprüche ausscheiden werden, wobei sich infolge der eher geringen Anzahl der betreffenden vor Gericht verfolgten Straftaten die finanziellen Auswirkungen für den Staat ohnehin in Grenzen halten werden. Für den einzelnen Menschen, der von der Gesetzesänderung betroffen wäre, ebenso wie für die Wahrheitsermittlung überwiegen jedenfalls die positiven Auswirkungen.

11 Der Mandantschaft ist nicht genützt, wenn die Zeugenaussage in Zweifel gezogen wird, was bei einem Auswendiglernen naheliegt. Korrektes Vorgehen der Nebenklagevertretung gefährdet eine Überprüfung der Konstanz der Aussage nicht.

12 Hierunter fallen etwa die Fälle, in denen das Opfer zur sexuellen Handlung durch Drohung mit einem ,sonstigen Übel“, also nicht mit „,gegenwärtiger Gefahr für Leib oder Leben“ (sonst: $§ 177$ StGB) genötigt wird, z.B. Tötung des Lieblingstiers, Verlust einer Arbeitsstelle oder Veranlassung der Rückschiebung in das Heimatland. 